\title{
Topological Behaviors in Networks of Time-Multiplexed Optical Resonators
}

\author{
Christian Leefmans ${ }^{1}$, Avik Dutt ${ }^{2}$, James Williams ${ }^{3}$, Luqi Yuan ${ }^{4}$, Shanhui Fan², \\ Alireza Marandi ${ }^{1}$ \\ ${ }^{1}$ Department of Electrical Engineering, California Institute of Technology. 1200 E California Blvd., Pasadena, \\ CA 91125, USA. \\ ${ }^{2}$ Department of Electrical Engineering, Stanford University, Stanford, CA 94305, USA. \\ ${ }^{3}$ Department of Electrical Engineering, Northwestern University, Evanston, IL 60208, USA. \\ ${ }^{4}$ School of Physics and Astronomy, Shanghai Jiao Tong University, Shanghai 200240, China \\ marandi@caltech.edu
}

\begin{abstract}
We observe topological phenomena in coupled, time-multiplexed optical resonators by mapping topologically nontrivial energy spectra to the network's loss. We detect topological edge states and reconstruct the band structures of noninteracting tight-binding Hamiltonians. (c) 2020 The Author(s)
\end{abstract}

Since Haldane and Raghu's observation that electromagnetic waves could exhibit topological behaviors [1], the field of topological photonics has developed rapidly on a variety of experimental platforms [2]. To overcome the fabrication and coupling limitations of spatially coupled structures, researchers have turned to synthetic dimensions to develop flexible and scalable architectures that implement a broad range of Hamiltonians [3]. Among synthetic dimension protocols, time-multiplexed optical resonators offer a promising, scalable platform with dense connectivity $[4,5]$. Here, we study the topological behaviors of a 50-pulse, time-multiplexed resonator network with 1D, nearest-neighbor coupling. In a departure from previous topological photonics experiments, which invariably map the eigenenergies of Hamiltonians to optical frequencies [2], we map these eigenenergies to the loss of our optical network to observe edge state characteristics and to perform band structure measurements.

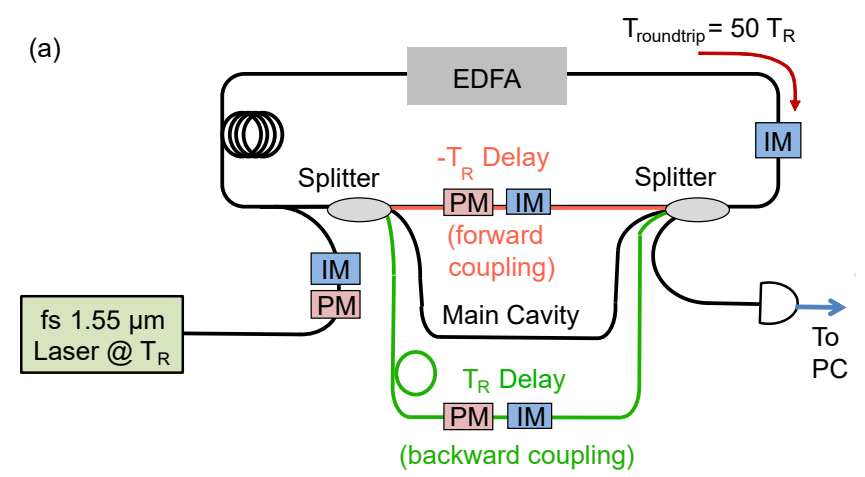

(b)

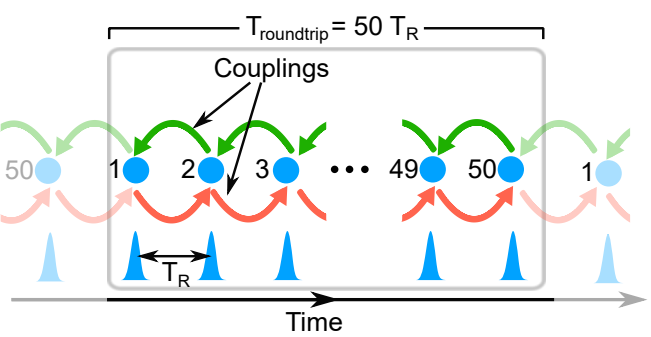

Fig. 1: (a) Schematic of the experiment. Our time-multiplexed optical resonator array consists of pulses itinerant in a fiber loop. The main cavity ( $\sim 40 \mathrm{~m}$ length) supports $\mathrm{N}=50$ pulses with a pulse period $T_{R}=4 \mathrm{~ns}$. The $+T_{R}$ and $-T_{R}$ delay lines are longer and shorter than the main cavity path by one pulse period and introduce bidirectional coupling between the $n^{\text {th }}$ and $(n \mp 1)^{\text {th }}$ pulses. The phase modulators (PM) and intensity modulators (IM) in the delay lines control the coupling strengths and phases, while a phase modulator and an intensity modulator prior to the cavity control the intensity and phase of the input light. The modulators before the cavity allow us to engineer pulse streams that correspond to eigenstates of Hamiltonians under study. (b) Illustration of the time-multiplexed 1D chain. Each pulse, represented with a blue dot, couples to its nearest neighbors through the optical delay lines. Using the intensity modulators in the delay lines, we can impose periodic boundary conditions or realize a finite lattice of up to 50 sites. This time-multiplexed scheme can be extended to larger lattices with potentially arbitrary couplings $[4,5]$.

The novel energy-to-loss mapping arises from the general differential equations for a system of coupled optical resonators, $i \dot{\vec{a}}=\mathscr{A} \vec{a}$. We identify the off-diagonal elements of $\mathscr{A}$ with a Hamiltonian, $\mathscr{H}$ and the diagonal elements with each resonator's loss. In the low-loss limit, the matrix equation is nothing but the Schrödinger equation, and the eigenenergies of the Hamiltonian map to the eigenfrequencies of the network. However, we can also introduce an additional $\pi / 2$ phase into each coupling to cancel the imaginary unit in our original equation. Then 
the energy of the Hamiltonian maps to the loss of the optical network.

We study this energy-to-loss map using the setup shown in Figure 1(a). This fiber optic network consists of a time-multiplexed optical resonators coupled through optical delay lines to simulate NN coupling in 1D tightbinding Hamiltonians. Figure 1(b) illustrates the couplings implemented by the network.

(a)

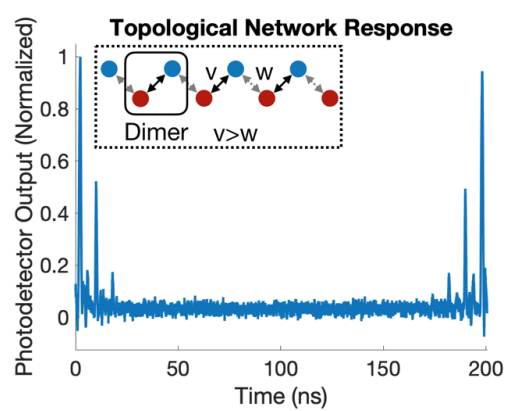

(b)

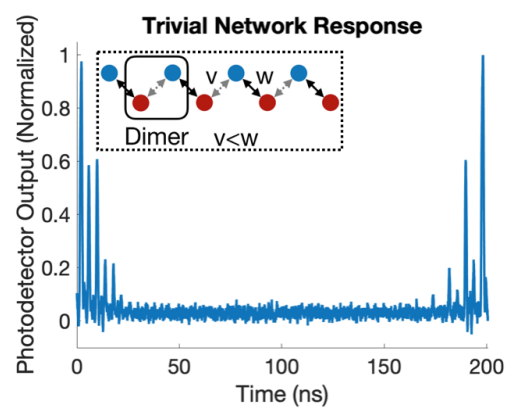

(c)

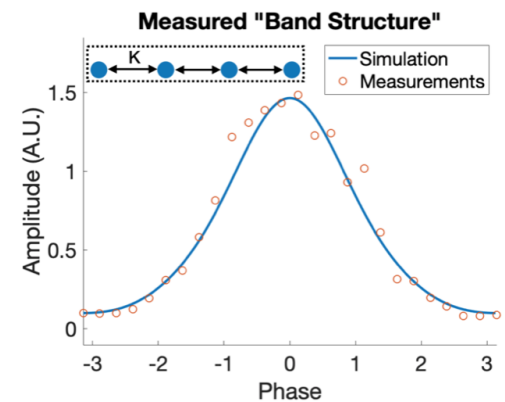

Fig. 2: (a) Topological edge state. The SSH model considers a 1D chain of dimers. When the inter-dimer coupling $(v)$ exceeds the intra-dimer coupling $(w)$, the model exhibits a topological edge state, while when $w$ exceeds $v$, the model is topologically trivial. The flexibility of the time-multiplexing scheme allows us to reprogram our couplings to achieve either phase. In the topological phase, we excite the edge state in a network corresponding to 25 dimers and observe that the light remains localized in the edge state. The alternating light and dark time slots are hallmarks of the SSH edge state. (b) Trivial edge state. We program the couplings between adjacent pulses to simulate the SSH model in the topologically trivial phase. When we excite the system with the edge state, we see that the light leaks into neighboring sites, which indicates that the system no longer exhibits the edge state. We believe that the asymmetry of this plot is due to an asymmetry in the coupling strengths between neighboring pulses. (c) Band structure measurement. We measure the band structure of a one-band model with real, nearest-neighbor coupling, $\kappa$. To measure points on the band structure, we excite the network with Bloch waves that correspond to different k-vectors in the Brillouin zone and measure the steady-state amplitude of the system. Because this steady-state amplitude is related inversely to the loss, we can map the measured amplitudes to the spectrum of the Hamiltonian. This same concept can be extended to more complicated Hamiltonians.

To demonstrate this energy-to-loss map, we observe the edge state of the Su-Schrieffer-Heeger (SSH) model and measure the band structure of a one-band model. Figures 2(a) and 2(b) demonstrate topologically nontrivial and trivial phases of the SSH model, respectively, while Figure 2(c) shows the band structure measurement of the one-band model.

In conclusion, we developed a 1D network of time-multiplexed optical resonators with nearest-neighbor coupling, and we observed signatures of the topologically nontrivial edge state of the SSH model. In addition, we reconstructed the band structure of a one-band model using a unique energy-to-loss map. The time-multiplexing scheme presented here potentially enables arbitrary coupling to study more complex models in high dimensions, as introducing new couplings and more dimensions simply corresponds to introducing new delay lines into the network shown in Figure 1(a). Hence, this architecture is a promising route to overcome the challenges faced by previous synthetic dimension schemes, which struggle to realize arbitrary long-range coupling and to simulate high dimensional physics [3].

This work is supported by ARO Grant No. W911NF-18-1-0285 and by NSF Grant No. 1846273

\section{References}

1. S. Raghu and F. Haldane, "Analogs of quantum-hall-effect edge states in photonic crystals," Phys. Rev. A 78, 033834 (2008).

2. T. Ozawa, et al., “Topological photonics,” Rev. Mod. Phys. 91, 015006 (2019).

3. L. Yuan, Q. Lin, M. Xiao, and S. Fan, "Synthetic dimension in photonics," Optica 5, 1396-1405 (2018).

4. A. Marandi et al., "Network of time-multiplexed optical parametric oscillators as a coherent ising machine," Nat. Photonics 8, 937-942 (2014).

5. P. McMahon et al., "A fully programmable 100-spin coherent ising machine with all-to-all connections," Science 354, 614-617 (2016). 